\title{
Medium effects on the rho meson
}

\author{
M. Asakawa and C. M. Ko \\ Cyclotron Institute and Physics Department, Texas AGM University, College Station, Texas 77843
}

(Received 23 March 1993)

\begin{abstract}
The property of a rho meson in dense nuclear matter is studied using the QCD sum rule. The spectral function appearing on the hadronic side of the sum rule is evaluated in the vector dominance model that takes into account the interaction between the rho meson and the pion. Including pion modification by the delta-hole polarization in the nuclear medium, we find that as the nuclear density increases the rho meson peak in the spectral function shifts to smaller invariant masses and its width becomes smaller. We discuss the possibility of studying the rho meson property in dense matter via the dilepton invariant mass spectrum from heavy-ion collisions.
\end{abstract}

PACS number(s): 25.75.+r, 14.40.Cs, $12.40 . \mathrm{Vv}$

We have recently studied the rho meson property in nuclear medium in the vector dominance model (VDM) $[1,2]$. Including the coupling of the rho meson to the pion, which is further modified by the delta-hole polarization of the medium, we find that with increasing nuclear density the position of the rho meson peak in the spectral function moves to larger invariant masses and its width increases [3]. Similar conclusions have also been obtained by Hermann et al. [4]. In Ref. [3], we have further found that the in-medium rho meson mass is, however, reduced if the bare rho meson mass in the model is assumed to decrease in the medium according to the scaling Ansatz of Brown and Rho [5]. We have thus concluded that the mean-field effect, parametrized by the scaling mass in Ref. [3], is more important than the loop corrections calculated by the VDM.

A more consistent way to incorporate the mean-field effect is through the QCD sum rule. In the QCD sum rule [6], the spectral function appearing in the dispersion relation for the current-current correlation function is usually taken to be a delta function plus a continuum. According to Hatsuda and Lee [7], the rho meson mass in the medium determined from the QCD sum rule decreases with increasing density as a result of the partial restoration of chiral symmetry in dense matter. To include loop corrections in the hadronic side, one can use the spectral function from the VDM but treat the bare rho meson mass as a parameter to be determined from the QCD sum rule. by

In terms of quarks, the current for a rho meson is given

$$
J_{\mu}=\frac{\bar{u} \gamma_{\mu} u-\bar{d} \gamma_{\mu} d}{2}
$$

Its correlation function in the medium

$$
\Pi_{\mu \nu}(q)=i \int e^{i q x}\left\langle T J_{\mu}(x) J_{\nu}(0)\right\rangle_{\rho} d^{4} x
$$

where $\langle\cdots\rangle_{\rho}$ denotes the expectation value in the medium, can be expressed in terms of the transverse and longitudinal parts. At zero momentum, $\mathbf{q}=0$, the two are, however, related and only the longitudinal correlation function $\Pi$ is needed.

The real and imaginary parts of the correlation function are related by the dispersion relation,

$$
\operatorname{Re} \Pi\left(Q^{2}\right)=\frac{P}{\pi} \int_{0}^{\infty} d s \frac{\operatorname{Im} \Pi(s)}{s+Q^{2}}+\text { subtractions. }
$$

For large Euclidean four-momenta, $Q^{2}\left(=-q^{2}=\right.$ $-s) \rightarrow \infty$, the real part can be evaluated perturbatively by the operator product expansion [8]. Including operators up to dimension 6 and twist 2 , we have

$$
\begin{aligned}
& \Pi\left(Q^{2}\right)=-\frac{1}{8 \pi^{2}}\left(1+\frac{\alpha_{s}}{\pi}\right) \ln \frac{Q^{2}}{Q_{0}^{2}}+\frac{m_{q}}{Q^{4}}\langle\bar{q} q\rangle_{\rho}+\frac{\alpha_{s}}{24 \pi Q^{4}}\left\langle G_{\mu \nu} G^{\mu \nu}\right\rangle_{\rho} \\
& -\frac{\pi \alpha_{s}}{Q^{6}}\left\langle\left(\bar{q} \gamma_{\mu} \gamma_{5} \lambda^{a} q\right)\left(\bar{q} \gamma^{\mu} \gamma_{5} \lambda^{a} q\right)\right\rangle_{\rho}-\frac{2 \pi \alpha_{s}}{9 Q^{6}} \sum_{q^{\prime}=u, d}\left\langle\left(\bar{q} \gamma_{\mu} \lambda^{a} q\right)\left(\bar{q}^{\prime} \gamma^{\mu} \lambda^{a} q^{\prime}\right)\right\rangle_{\rho} \\
& -\frac{2 i}{3} \frac{q_{\sigma_{1}} q_{\sigma_{2}}}{Q^{6}} \sum_{q^{\prime}=u, d}\left\langle\mathcal{S} \bar{q}^{\prime} \gamma^{\sigma_{1}} D^{\sigma_{2}} q^{\prime}\right\rangle_{\rho}+\frac{8 i}{3} \frac{q_{\sigma_{1}} q_{\sigma_{2}} q_{\sigma_{3}} q_{\sigma_{4}}}{Q^{10}} \sum_{q^{\prime}=u, d}\left\langle\mathcal{S} \bar{q}^{\prime} \gamma^{\sigma_{1}} D^{\sigma_{2}} D^{\sigma_{3}} D^{\sigma_{4}} q^{\prime}\right\rangle_{\rho}
\end{aligned}
$$

In the above, $\alpha_{s}$ is the QCD coupling constant, $Q_{0}$ is an arbitrary scale parameter, and $D_{\mu}=\partial_{\mu}-i g A_{\mu}^{a} \lambda^{a} / 2$ with $\lambda^{a}$ the $\mathrm{SU}(3)$-color matrices and $A_{\mu}^{a}$ the gluon field. Both the light quark masses and their condensates are taken to be the same, i.e., $m_{q}=m_{u}=m_{d}$ and $\langle\bar{q} q\rangle_{\rho}=\langle\bar{u} u\rangle_{\rho}=\langle\bar{d} d\rangle_{\rho}$. The last two terms in the above equation are the derivative condensates from the nonscalar operators as a result of the breaking of Lorentz invariance in the medium $[7,9]$. The symmetrization and traceless operator is denoted by $\mathcal{S}$. 
The imaginary part of the correlation function at $s>$ 0 is parametrized phenomenologically by a contribution from the rho meson and a continuum, i.e.,

$8 \pi \operatorname{Im} \Pi(s)=F \frac{S(s)}{s} \theta\left(s_{0}-s\right)+\left(1+\frac{\alpha_{s}}{\pi}\right) \theta\left(s-s_{0}\right)$.

In the above, $F$ is a constant, $s_{0}$ is the continuum threshold, and $S(s)$ is the rho meson spectral function. The factor $1 / s$ is required to relate the imaginary part of the correlation function to the spectral function in hadronic models.

In the normal QCD sum rule [7], the rho meson spectral function is taken to be a delta function, i.e., $\delta\left(s-m_{\rho}^{2}\right)$. Here we shall use the one from the vector dominance model that includes the effect from the delta-hole polarization, i.e.,

$$
S(s)=-\frac{2 \Sigma_{I}(s)}{\left[s-m_{\rho}^{2}-\Sigma_{R}(s)\right]^{2}+\left[\Sigma_{I}(s)\right]^{2}},
$$

where $\Sigma_{R}$ and $\Sigma_{I}$ are, respectively, the renormalized real and imaginary parts of the rho meson self-energy as cal- culated in Ref. [3]. The bare rho meson mass $m_{\rho}$ is about $770 \mathrm{MeV}$ in free space but becomes density dependent in the medium due to the change of the quark condensate.

To suppress the contribution from higher-order operators, one usually introduces the Borel transform which is defined by

$L_{M}=\lim _{Q^{2}, n \rightarrow \infty ; Q^{2} / n=M^{2}} \frac{1}{(n-1) !}\left(Q^{2}\right)^{n}\left(-\frac{d}{d Q^{2}}\right)^{n}$,

where $M$ is the Borel mass. The Borel transform also removes the need for subtractions in the dispersion relation.

Carrying out the Borel transform of both sides of Eq. (3) and taking the ratio of the resulting equation to its derivative with respect to $-1 / M^{2}$, we obtain

$$
\frac{\int_{0}^{s_{0}} e^{-s / M^{2}} S(s) d s}{\int_{0}^{s_{0}} e^{-s / M^{2}}[S(s) / s] d s}=\frac{N}{D}
$$

where

$$
\begin{aligned}
N=M^{2}\{ & \left(1+\frac{\alpha_{s}}{\pi}\right)\left[1-\left(1+\frac{s_{0}}{M^{2}}\right) e^{-s_{0} / M^{2}}\right]-\frac{8 \pi^{2} m_{q}}{M^{4}}\langle\bar{q} q\rangle_{\rho} \\
& \left.\quad-\frac{\pi^{2}}{3 M^{4}}\left\langle\frac{\alpha_{s}}{\pi} G^{2}\right\rangle_{\rho}+\frac{896 \pi^{3} \alpha_{s}}{81 M^{6}}\langle\bar{q} q\rangle_{\rho}^{2}-\frac{2 \pi^{2}}{M^{4}} A_{1} m_{N} \rho_{N}+\frac{10 \pi^{2}}{3 M^{6}} A_{3} m_{N}^{3} \rho_{N}\right\}
\end{aligned}
$$

and

$$
D=\left(1+\frac{\alpha_{s}}{\pi}\right)\left(1-e^{-s_{0} / M^{2}}\right)+\frac{8 \pi^{2} m_{q}}{M^{4}}\langle\bar{q} q\rangle_{\rho}+\frac{\pi^{2}}{3 M^{4}}\left\langle\frac{\alpha_{s}}{\pi} G^{2}\right\rangle_{\rho}-\frac{448 \pi^{3} \alpha_{s}}{81 M^{6}}\langle\bar{q} q\rangle_{\rho}^{2}+\frac{2 \pi^{2}}{M^{4}} A_{1} m_{N} \rho_{N}-\frac{5 \pi^{2}}{3 M^{6}} A_{3} m_{N}^{3} \rho_{N}
$$

In deriving Eq. (8), we have made use of the usual meanfield approximation,

$$
\begin{aligned}
\left\langle\left(\bar{q} \gamma_{\mu} \gamma_{5} \lambda^{a} q\right)\left(\bar{q} \gamma^{\mu} \gamma_{5} \lambda^{a} q\right)\right\rangle_{\rho} & \approx \frac{16}{9}\langle\bar{q} q\rangle_{\rho}^{2} \\
\left\langle\left(\bar{q} \gamma_{\mu} \lambda^{a} q\right)\left(\bar{q}^{\prime} \gamma^{\mu} \lambda^{a} q^{\prime}\right)\right\rangle_{\rho} & \approx-\frac{16}{9}\langle\bar{q} q\rangle_{\rho}^{2} \delta_{q q^{\prime}}
\end{aligned}
$$

The vacuum condensates, quark mass, and QCD coupling constant are taken to be $\langle\bar{q} q\rangle_{0}=-0.023 \mathrm{GeV}^{3}$, $\frac{\alpha_{s}}{\pi}\left\langle G_{\mu \nu} G^{\mu \nu}\right\rangle_{0}=0.0168 \mathrm{GeV}^{4}, m_{q}=3.6 \mathrm{MeV}$, and $\alpha_{s}=$ 0.3. All except the quark condensate are commonly adopted values. We use a smaller value for the quark condensate than that of Ref. [7] in order to get the correct rho meson mass in free space with our imaginary part of the correlation function.

The in-medium scalar condensates are smaller in absolute value than the vacuum ones as nucleons remove condensates in the vacuum. For not too high nuclear densities, the density dependence of the in-medium condensates can be approximately expressed as

$$
\begin{aligned}
\langle\bar{q} q\rangle_{\rho} & \approx\langle\bar{q} q\rangle_{0}+\langle\bar{q} q\rangle_{N} \rho_{N} \\
\frac{\alpha_{s}}{\pi}\left\langle G_{\mu \nu} G^{\mu \nu}\right\rangle_{\rho} & \approx \frac{\alpha_{s}}{\pi}\left\langle G_{\mu \nu} G^{\mu \nu}\right\rangle_{0}-\frac{8}{9} m_{N}^{0} \rho_{N}
\end{aligned}
$$

where $m_{N}^{0}=830 \mathrm{MeV}$ is the nucleon mass in the chiral limit [7], $\rho_{N}$ is the density of the medium, and $\langle\bar{q} q\rangle_{N}$ is the quark content of the nucleon. The second relation follows from the trace anomaly in QCD [7].

The derivative condensates in Eq. (4) are approximately given by the product of the nuclear density and the expectation value of the corresponding operators in the nucleon. The latter can be expressed in terms of the moments of the quark distribution in a nucleon [10], i.e.,

$$
\begin{aligned}
& \sum_{q^{\prime}=u, d}\left\langle\mathcal{S} \bar{q}^{\prime} \gamma_{\mu_{1}} D_{\mu_{2}} \cdots D_{\mu_{n}} q^{\prime}\right\rangle_{N} \\
&=(-i)^{n-1} A_{n-1}\left(\frac{1}{2 m_{N}} p_{\mu_{1}} \cdots p_{\mu_{n}}-\text { trace terms }\right), \\
& \text { for } n=\text { even. }
\end{aligned}
$$

In the above, $p_{\mu}$ is the momentum of the nucleon and

$$
A_{n}=2 \int_{0}^{1} x^{n}\left[u_{N}(x)+\bar{u}_{N}(x)+d_{N}(x)+\bar{d}_{N}(x)\right] d x .
$$

Using the empirically known quark distribution function in a nucleon at the QCD perturbative scale of $1 \mathrm{GeV}$ [11], we find that $A_{1} \approx 0.938$ and $A_{3} \approx 0.121$.

To determine the rho meson mass $m_{\rho}^{*}$ in the nuclear 
medium, we solve the sum rule in two steps. We first fix the threshold energy $s_{0}$ by requiring that $N / D$ on the right-hand side of the sum rule, Eq. (8), is insensitive to the Borel mass in the range of $0.55 \leq M^{2} \leq 0.75 \mathrm{GeV}^{2}$. Then the bare rho meson mass $m_{\rho}$ in the spectral function $S(s)$ is determined by equating the left-hand side of the sum rule, Eq. (8), to the determined $N / D$. This procedure is found to work very well. We note that for $S(s)=\delta\left(s-m_{\rho}^{2}\right)$ as in the normal QCD sum rule the square of the rho meson mass is simply given by $N / D$.

We find that the bare rho meson mass $m_{\rho}$ decreases with increasing nuclear density as a result of the reduced quark condensate in the medium. The in-medium rho meson mass $m_{\rho}^{*}$, determined by the pole of the rho meson propagator, i.e., $F\left(m_{\rho}^{*}\right)=m_{\rho}^{* 2}-m_{\rho}^{2}-\Sigma_{R}=0$, is shown by the solid curve in Fig. 1 . It is about $530 \mathrm{MeV}$ and 220 $\mathrm{MeV}$ at normal and twice normal nuclear matter density, respectively. The corresponding continuum threshold $s_{0}$ is about $0.87 \mathrm{GeV}^{2}$ and $0.47 \mathrm{GeV}^{2}$, respectively. We have also shown in this figure by the dotted curve the in-medium rho meson mass determined from the usual QCD sum rule using a delta function for the rho meson spectral function [7]. We see that our results are similar to those from the normal QCD sum rule calculation.

The rho meson spectral function in the medium, given by Eq. (6) and evaluated with the density-dependent rho meson bare mass, is shown in Fig. 2. We see that as the peak of the spectral function moves to smaller invariant masses, $M=s^{1 / 2}$, its width also becomes smaller. These results are qualitatively similar to those of Ref. [3] using the scaling rho meson mass in the vector dominance model.

In our study, we have neglected in the operator product expansion the twist-4 operators such as $\left(\bar{q} \gamma_{\mu} q \bar{q} \gamma_{\nu} q\right),\left(\bar{q} \gamma_{\mu} \gamma_{5} q \bar{q} \gamma_{\nu} \gamma_{5} q\right),\left(\bar{q} \gamma^{\lambda}\left[D_{\mu}, G_{\lambda \nu}\right] q\right)$, and $\left(\bar{q} \gamma^{\lambda} \gamma_{5}\left\{D_{\mu}, \tilde{G}_{\lambda \nu}\right\} q\right)$ with $\tilde{G}_{\mu \nu}=\epsilon_{\mu \nu \sigma \rho} G^{\sigma \rho} / 2$ as the density dependence of these condensates is not known. We do not know at present if our result will be modified by these operators. Further studies are thus needed.

Also, the accuracy of the mean-field approximation

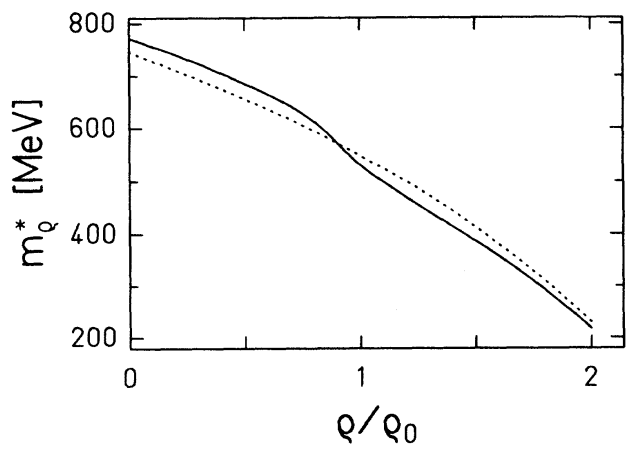

FIG. 1. The density dependence of the rho meson mass. The solid curve is obtained with the rho meson spectral function evaluated in the vector dominance model that includes the delta-hole polarization of the nuclear medium. The dotted curve is from the usual QCD sum rule calculation assuming that the rho meson spectral function is a delta function.

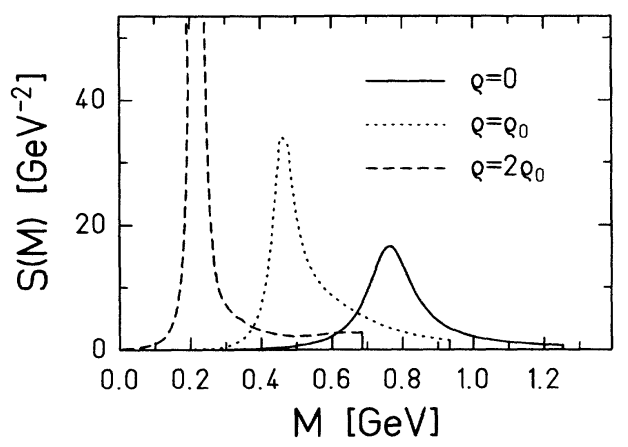

FIG. 2. The spectral function of a rho meson. The solid curve is for a rho meson in free space. For a rho meson in the medium, the dotted and dashed curves correspond, respectively, to nuclear densities of $\rho_{0}$ and $2 \rho_{0}$, where $\rho_{0}$ is the normal nuclear matter density.

used in Eq. (11) to relate the 4-quark condensates to the 2-quark condensate in the medium is not known. Therefore, there remains the possibility that the density dependence of the 4-quark condensates is much weaker than Eqs. (11) and (12). In this case, we expect that the QCD sum rule will be satisfied with a much smaller mass shift of the rho meson. Indeed, if we assume that the 4-quark condensates do not depend on the density and are given by their vacuum values determined from the mean-field approximation, the in-medium rho meson mass is found almost independent of the density. The rho spectral function in the medium is therefore similar to that given by the vector dominance model $[3,4]$.

In conclusion, we have introduced a consistent method to incorporate both the mean-field effect and the loop corrections on the rho meson property in dense nuclear matter. This is achieved by using the spectral function calculated from the vector dominance model in the hadronic side of the QCD sum rule. The in-medium rho meson mass is found to decrease in the matter if we assume that the density dependence of the 4-quark condensates can be factorized as the square of the 2-quark condensate. This confirms our previous results based on the scaling mass that the mean-field effect is more important than the loop corrections.

The property of a rho meson in dense matter can be studied via dilepton production from the pion-pion annihilation in heavy-ion collisions [12-18]. Although the reduced rho meson mass in the medium makes its identification more difficult because of the background from eta decay and bremsstrahlungs [19], heavy-ion experiments that are being carried out at the Bevalac $[20,21]$ and being planned at the Heavy-Ion Synchrotron at GSI offer a unique opportunity to verify experimentally the predicted property of the rho meson in dense nuclear matter.

This work was supported in part by the National Science Foundation under Grant No. PHY-9212209 and the Welch Foundation under Grant No. A-1110. 
[1] J. J. Sakurai, Currents and Mesons (University of Chicago Press, Chicago, 1969)

[2] C. Gale and J. Kapusta, Nucl. Phys. B357, 65 (1991).

[3] M. Asakawa, C. M. Ko, P. Lévai, and X. J. Qiu, Phys. Rev. C 46, R1159 (1992).

[4] M. Hermann, B. L. Friman, and W. Nörenberg, Z. Phys. A343, 119 (1992).

[5] G. E. Brown and M. Rho, Phys. Rev. Lett. 66, 2720 (1991).

[6] M. A. Shifman, A. I. Vainstein, and V. I. Zakharov, Nucl. Phys. B147, 385 (1979).

[7] T. Hatsuda and S. H. Lee, Phys. Rev. C 46, R34 (1992).

[8] L. J. Reinders, H. Rubinstein, and S. Yazaki, Phys. Rep. 127, 1 (1985).

[9] E. V. Shuryak, Nucl. Phys. A544, 65c (1992).

[10] N. Christ, B. Hasslacher, and A. H. Mueller, Phys. Rev. D 6, 3543 (1972).

[11] M. Glück, E. Reya, and A. Vogt, Z. Phys. C 48, 471 (1990).
[12] C. Gale and J. Kapusta, Phys. Rev. C 35, 2107 (1987).

[13] L. H. Xia, C. M. Ko, L. Xiong, and J. Q. Wu, Nucl. Phys. A485, 721 (1988).

[14] C. M. Ko, L. H. Xia, and P. J. Siemens, Phys. Lett. B 231, 16 (1989).

[15] C. L. Korpa and S. Pratt, Phys. Rev. Lett. 64, 1502 (1990).

[16] C. L. Korpa, L. Xiong, C. M. Ko, and P. J. Siemens, Phys. Lett. B 246, 333 (1990).

[17] L. Xiong, Z. G. Wu, C. M. Ko, and J. Q. Wu, Nucl. Phys. A512, 772 (1990).

[18] Gy. Wolf, G. Batko, T. S. Biro, W. Cassing, and U. Mosel, Nucl. Phys. A517, 615 (1990).

[19] Gy. Wolf, W. Cassing, and U. Mosel, Nucl. Phys. A552, 549 (1993).

[20] G. Roche et al., Phys. Lett. B 226, 228 (1989); Phys. Rev. Lett. 61, 1069 (1989).

[21] C. Naudet et al., Phys. Rev. Lett. 62, 2652 (1989). 